\title{
Giant triplet proximity effect in superconducting pseudo spin valves with engineered anisotropy
}

\author{
X. L. Wang, ${ }^{1,2}$ A. Di Bernardo, ${ }^{1}$ N. Banerjee,${ }^{1}$ A. Wells, ${ }^{1}$ F. S. Bergeret,${ }^{3}$ M. G. Blamire, ${ }^{1}$ and J. W. A. Robinson ${ }^{1, *}$ \\ ${ }^{1}$ Department of Materials Science and Metallurgy, University of Cambridge, 27 Charles Babbage Road, \\ Cambridge CB3 OFS, United Kingdom \\ ${ }^{2}$ State Key Laboratory of Superlattices and Microstructures, Institute of Semiconductors, Chinese Academy of Sciences, Beijing 100083, China \\ ${ }^{3}$ Centro de Física de Materiales (CFM-MPC), Centro Mixto CSIC-UPV/EHU, Manuel de Lardizabal 4, E-20018 San Sebastián, Spain
}

(Received 6 March 2014; revised manuscript received 4 April 2014; published 30 April 2014)

\begin{abstract}
The proximity coupling of a thin superconducting layer and an inhomogeneous ferromagnet can lead to a significant reduction of the critical temperature due to the generation of spin-polarized triplet Cooper pairs. We report critical temperature measurements of $\mathrm{Co} / \mathrm{Cu} / \mathrm{NiFe}(\mathrm{Py}) / \mathrm{Cu} / \mathrm{Nb}$ superconducting pseudo spin valves (PSVs) in which the magnetization of the soft layer (Py) can be independently rotated in-plane with a magnetic field to create an angle $(\theta)$ between it and the magnetization of Co. Here we observe results consistent with spin-triplet theory and demonstrate large changes in $\Delta T_{C}$ up to $-120 \mathrm{mK}$ as the Py layer is rotated from $0^{\circ}$ (Co and Py are parallel) to $90^{\circ}$ (Co and Py are orthogonal), which offers the potential for active control of the superconducting state. The key to this achievement is engineered magnetic anisotropy in Py, which enables well-defined control over the magnetization configuration of the PSV.
\end{abstract}

DOI: 10.1103/PhysRevB.89.140508

PACS number(s): 85.25.Cp, 74.50.+r

Spintronics was initiated following the discovery of giant magnetoresistance (GMR) in magnetic heterostructures [1]. A classic GMR structure consists of ferromagnetic/ nonmagnetic/ferromagnetic (F-N-F) layers in which the differential scattering of electron spins results in the electrical resistance $(R)$ depending on the mutual orientation of the $\mathrm{F}$ layer moments: When the F layers are antiparallel (AP) the majority and minority electron spins are scattered meaning $R$ is maximized, but when the $\mathrm{F}$ layers are parallel $(\mathrm{P})$ only the minority spins are scattered and so $R$ is minimized. The aim of spintronics is to create logic circuits which exploit spindependent scattering and the polarization of spin-currents, to process information with the promise of higher operating frequencies than in conventional charge-based electronics.

Although superconducting materials are attractive for spintronic circuits as interconnects for transmitting spinpolarized (nonsuperconducting) quasiparticle currents [2,3], the real breakthrough would be the creation of devices which exploit the spin of triplet Cooper pairs [4]. Spin-polarized triplet Cooper pairs are generated at superconductor/F (S-F) interfaces via a spin-mixing process $[5,6]$. The key to this process is magnetic inhomogeneity at the S-F interface. For example, consider a S-F'-F-F'-S Josephson junction [7]: If the $\mathrm{F}^{\prime}$ and $\mathrm{F}$ layer magnetizations are collinearly aligned, spin-zero triplet pairs form which, like singlet pairs, are short ranged [8] with a coherence length in F metals of only a few nanometers (Ni [9-12], NiFe [13,14], Co [11,15,16], and Fe [17,18]). However, if $\mathrm{F}^{\prime}$ and $\mathrm{F}$ layer magnetizations are noncollinearly aligned spin-one triplet pairs form and the coherence length is greatly extended [19-27]. See also spectroscopic works on triplet S-F structures in Refs. [28-30].

The fundamental premise of superconducting spintronics is that active devices can be created in which triplet supercurrents, instead of nonsuperconducting spin-currents, control the electronic state of circuit components. Theory has already gone some way in developing this field with the demonstration

*jjr33@cam.ac.uk that triplet pairs can apply spin transfer torque to magnetic nanoelements [31,32]. To develop practical devices, optimized spin-mixers for generating large triplet spin-current densities are required and the net spin that can be generated by a triplet state should be quantified.

As shown theoretically by Fominov et al. [33], critical temperature $\left(T_{C}\right)$ measurements of S-F1-F2 pseudo spin valves (PSVs) are attractive for investigating the effect of magnetization alignment on the triplet proximity effect. In such superconducting PSVs singlet-to-spin-one-triplet pair conversion is maximized when F1 and F2 layer magnetizations are bi-quadratically coupled. If the $\mathrm{S}$ layer is thinner than BCS coherence length ( $35 \mathrm{~nm}$ in $\mathrm{Nb}$ ), pair conversion is manifested as a gradual decrease in $T_{C}$ as the angle $(\theta)$ between $\mathrm{F} 1$ and F2 increases to $90^{\circ}$. The $T_{C}$ decreases since the PSV serves as a sink for triplet pairs and so the loss of pairs from $S$ results in a reduction of the pairing amplitude (and therefore $T_{C}$ ).

In this paper we report $T_{C}$ measurements of $\mathrm{Co} / \mathrm{Cu} / \mathrm{Py} /$ $\mathrm{Cu} / \mathrm{Nb}$ PSVs as a function of angle $(\theta)$ between the Co and Py (NiFe) layer magnetizations. The Py layer is magnetically soft and anisotropic with a well-defined in-plane easy axis, while the Co layer is magnetically harder than Py and isotropic (in-plane). The magnetic anisotropy of Py is key since it means the magnetic state of the PSV is controllable. By independently rotating the Py layer magnetization with respect to $\mathrm{Co}$, we observe $\Delta T_{C}=T_{C}\left(0^{\circ}\right)-T_{C}\left(90^{\circ}\right)$ values as large as $-120 \mathrm{mK}$.

Leksin et al. [34] reported $T_{C}$ measurements on $\mathrm{CoO}_{x} / \mathrm{Fe} 1 / \mathrm{Cu} / \mathrm{Fe} 2 / \mathrm{Pb} \mathrm{SVs}$ in which $\Delta T_{C}=T_{C}\left(0^{\circ}\right)-T_{C}\left(90^{\circ}\right)$ values as large as $-50 \mathrm{mK}$ were obtained. In their experiment the antiferromagnetic layer $\left(\mathrm{CoO}_{X}\right)$ exchange biases Fe1 [34], which induces anisotropy in this pinned layer, while the Fe2 free layer is magnetically isotropic.

PSVs were deposited by dc magnetron sputtering onto unheated oxidized (100) silicon substrates in an ultrahigh vacuum deposition chamber. The chamber was cooled via a liquid nitrogen jacket to achieve a base pressure better than $5 \times 10^{-10}$ mbar. The different layers were grown in $1.5 \mathrm{~Pa}$ of $\mathrm{Ar}$ in series by passing the substrates below stationary magnetrons. Growth rates were precalibrated by atomic force 

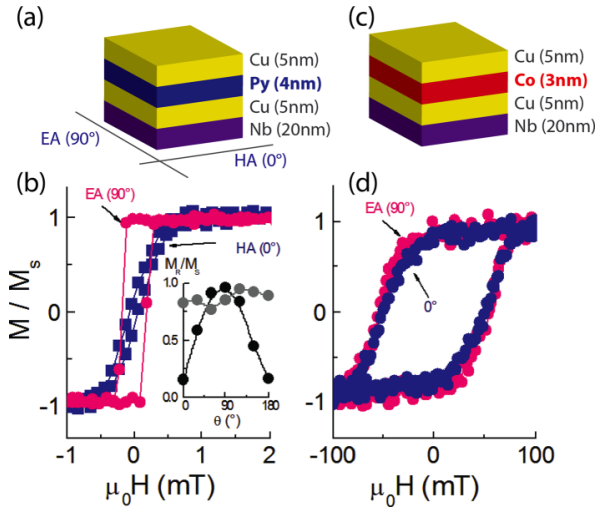

FIG. 1. (Color online) Illustrations of (a) $\mathrm{Cu} / \mathrm{Py} / \mathrm{Cu} / \mathrm{Nb}$ and (c) $\mathrm{Cu} / \mathrm{Co} / \mathrm{Cu} / \mathrm{Nb}$ control samples showing the orientation of the magnetic field applied during growth $\left(H_{\text {set }}\right)$ and the corresponding easy axis (EA) and hard axis (HA) of Py. (b) In-plane $M-H$ loops of the $\mathrm{Cu} / \mathrm{Co} / \mathrm{Cu} / \mathrm{Nb}$ structures in which the magnetic field is applied along the EA (pink) and HA (blue) of Py. The inset of (b) shows the angular dependence of the $M_{R} / M_{S}$ ratio at $300 \mathrm{~K}$ for the $\mathrm{Cu} / \mathrm{Py} / \mathrm{Cu} / \mathrm{Nb}$ (black, labeled $\mathrm{Py}$ ) and $\mathrm{Cu} / \mathrm{Co} / \mathrm{Cu} / \mathrm{Nb}$ (gray, labeled $\mathrm{Co}$ ) structures. (d) In-plane $M-H$ loops of the $\mathrm{Cu} / \mathrm{Co} / \mathrm{Cu} / \mathrm{Nb}$ structure.

microscopy on step-edges created by partial lift-off of thin films deposited on patterned substrates. The growth rate of the different layers was controlled by the target power and the speed in which the substrates passed below stationary targets.

To set the magnetic easy axis (EA) and hard axis (HA) in Py, the entire stacks were deposited in a constant magnetic field $\left(H_{\text {set }}\right)$ of $\sim 70-80 \mathrm{mT}$. Control samples of $\mathrm{Cu} / \mathrm{Py} / \mathrm{Cu} / \mathrm{Nb}$ and $\mathrm{Cu} / \mathrm{Co} / \mathrm{Cu} / \mathrm{Nb}$ were first prepared (Fig. 1) in order to characterize the magnetic properties of Py and Co. In-plane magnetization vs applied field $(M-H)$ loops of the control samples were acquired at room temperature using a vibrating sample magnetometer. Figure 1(b) shows $M-H$ loops from the $\mathrm{Cu} / \mathrm{Py} / \mathrm{Cu} / \mathrm{Nb}$ sample in which the applied field $H_{a}$ is directed at different angles: At $90^{\circ} H_{a}$ is parallel to the set field during growth and since the $M-H$ loop is square, meaning the ratio of the remanent magnetization to the saturation magnetization $\left(M_{R} / M_{S}\right)$ is close to $1, H_{a}$ is parallel to the EA; conversely, at $0^{\circ}, H_{a}$ is perpendicular to the set field during growth and $M_{R} / M_{S} \ll 1$ implying a HA orientation. This is confirmed in the inset of Fig. 1(b), which shows $M_{R} / M_{S}$ reaching a maximum value at $90^{\circ}$. Similar measurements on the $\mathrm{Cu} / \mathrm{Co} / \mathrm{Cu} / \mathrm{Nb}$ control sample [two $\mathrm{M}-\mathrm{H}$ loops are shown in Fig. 1(d)] show no dependence of $M_{R} / M_{S}$ on the applied field angle meaning the magnetization of $\mathrm{Co}$ is isotropic and unaffected by the set field applied during growth.

Once the magnetic anisotropy of Py was confirmed, we prepared $\mathrm{Cu} / \mathrm{Co}(3) / \mathrm{Cu} / \mathrm{Py}(4) / \mathrm{Cu} / \mathrm{Nb}$ (numbers in $\mathrm{nm}$ units) PSVs. In Fig. 2 we have plotted a typical in-plane $M-H$ loop of a PSV at $10 \mathrm{~K}$; the magnetic field was applied parallel to the EA of Py. The $M-H$ loop shows that beyond $\pm 50 \mathrm{mT}$ the PSV has P state and as the magnetic field direction is reversed, the Py (soft) layer switches around $\pm 3.5 \mathrm{mT}$ and a stable AP state is obtained in both the forward (negative to positive) and reverse field sweep directions. Upon increasing the field, the Co layer slowly switches over a broad field range of $10-45 \mathrm{mT}$ and the PSV returns to the P state.

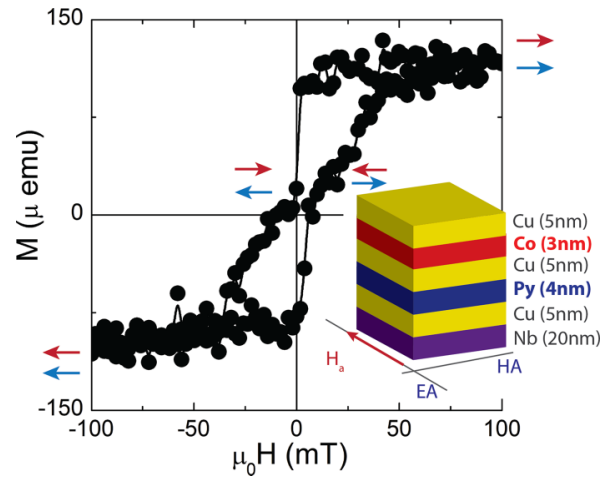

FIG. 2. (Color online) In-plane $M-H$ loop of a $\mathrm{Cu} / \mathrm{Co} / \mathrm{Cu} / \mathrm{Py} / \mathrm{Cu} / \mathrm{Nb} \mathrm{PSV}$ at $10 \mathrm{~K}$. Arrows indicate the likely magnetization configuration of the Co and Py layers at different points in the $M-H$ loop. Inset: Illustration of the PSV with the HA and EA of Py labeled and also the direction of the applied field $\left(H_{a}\right)$. The slight horizontal shift in field axis is a measurement artifact.

Resistance vs temperature $R(T)$ measurements of unpatterned PSVs was performed at temperatures around the superconducting transition (2-10 K) using a four-point current-bias $(100 \mu \mathrm{A})$ technique in a pulse-tube cryogen-free measurement system. We were careful to ensure that the superconducting transition was independent of the applied current. The $T_{C}$ was defined as the temperature corresponding to $50 \%$ of the resistive transition.

The effect of magnetization configuration on the $T_{C}$ of the PSVs was investigated by measuring $R(T)$ with a constant magnetic field applied in-plane at different angles $(\theta)$ with respect to the HA of Py (defined as $0^{\circ}$ ). This was achieved using the following procedure. (1) The PSV was magnetically saturated at $10 \mathrm{~K}$ with an in-plane field of $300 \mathrm{mT}$ along the HA of Py. The field was then reduced to zero. (2) The PSV was rotated to the desired value of $\theta$ with respect to the HA of Py. A constant in-plane field of $5 \mathrm{mT}$ was applied and $R(T)$ was measured by cooling at least $1 \mathrm{~K}$ below $T_{C}$ and then by warming back to $10 \mathrm{~K}$. (3) The PSV was rotated (without switching off the magnetic field) to a new value of $\theta$ and $R(T)$ was remeasured (in cooling and warming). (4) Step 3 was repeated multiple times so the $T_{C}$ as a function of $\theta$ could be obtained. A field of $5 \mathrm{mT}$ was sufficient to align and saturate the Py layer magnetization (at all values of $\theta$ ) without affecting Co, which remained fixed in a remanent state (see Fig. 2).

In Fig. 3 we have plotted $R(T)$ curves and extrapolated values of $T_{C}$ as a function of $\theta$ for two PSVs: (PSV-A) $\mathrm{Cu}(5) / \mathrm{Co}(6) / \mathrm{Cu}(5) / \mathrm{Py}(4) / \mathrm{Cu}(5) / \mathrm{Nb}(21)$ and ( $\mathrm{PSV}-\mathrm{B}$ ) $\mathrm{Cu}(5) / \mathrm{Co}(3) / \mathrm{Cu}(5) / \mathrm{Py}(4) / \mathrm{Cu}(5) / \mathrm{Nb}(20)$ (numbers in $\mathrm{nm}$ units). Two important features are observable. First, $T_{C}$ depends nonmonotonically on $\theta$ with a clear minimum in $T_{C}$ around $\theta=90^{\circ}\left(H_{a}\right.$ parallel to the EA of Py). The maximum change in $T_{C}$ between $\theta=0^{\circ}$ and $\theta=90^{\circ}\left(\Delta T_{C}\right)$ is roughly $-20 \mathrm{mK}$ for PSV-A and roughly $-120 \mathrm{mK}$ for PSV-B, which has a thinner layer of $\mathrm{Nb}(20 \mathrm{~nm})$. Second, for PSV-A the $T_{C}$ is greater when the Co and Py layer magnetizations are parallel than when they are antiparallel, while for PSV-B there was no noticeable difference between $T_{C}(\mathrm{P})$ and $T_{C}(\mathrm{AP})$. We note that the dependence of $T_{C}$ of a bare 20-nm-thick $\mathrm{Nb}$ film on 

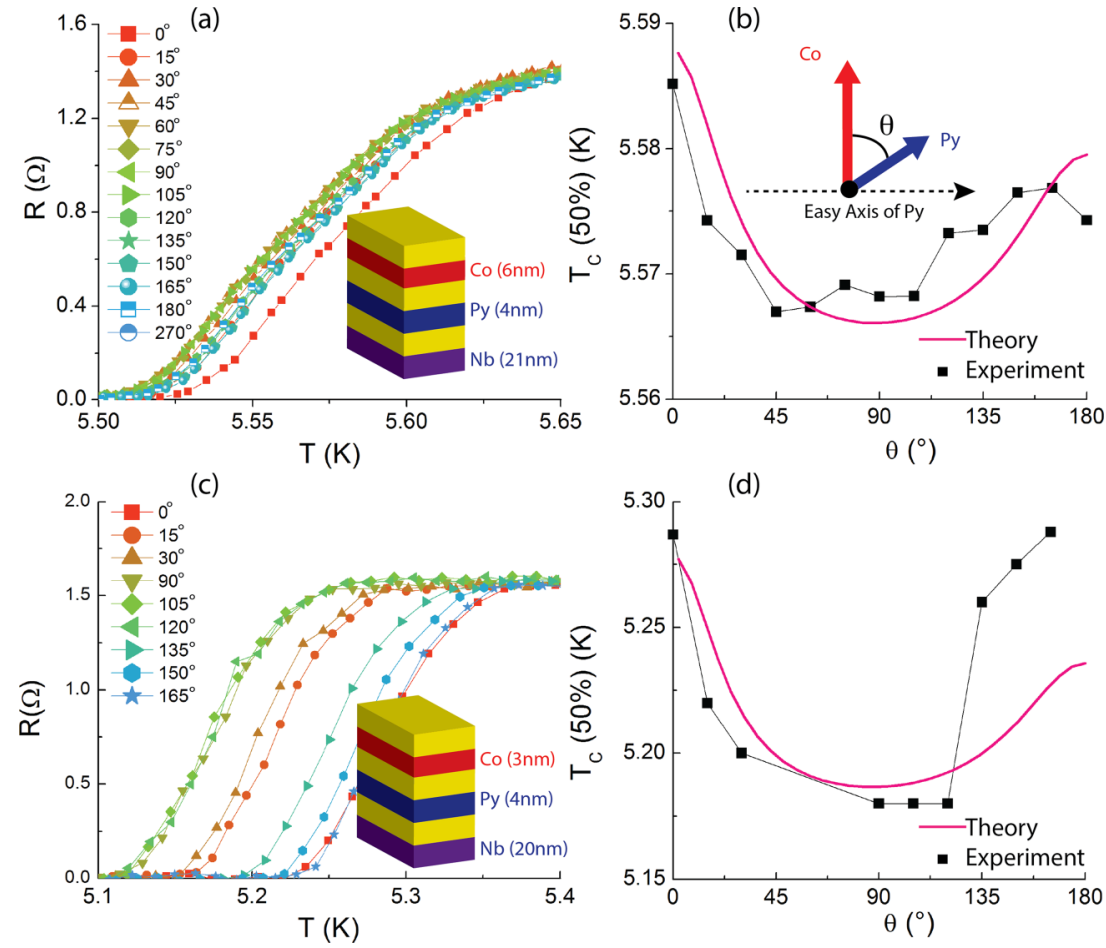

FIG. 3. (Color online) (a) and (c) $R(T)$ and (b) and (d) values of $T_{C}$ as a function of $\theta$ for (a) and (b) $\mathrm{Cu}(5 \mathrm{~nm}) / \mathrm{Co}(6 \mathrm{~nm}) / \mathrm{Cu}(5 \mathrm{~nm}) /$ $\mathrm{Py}(4 \mathrm{~nm}) / \mathrm{Cu}(5 \mathrm{~nm}) / \mathrm{Nb}(21 \mathrm{~nm})(\mathrm{PSV}-\mathrm{A})$ and $(\mathrm{c})$ and (d) $\mathrm{Cu}(5 \mathrm{~nm}) / \mathrm{Co}(3 \mathrm{~nm}) / \mathrm{Cu}(5 \mathrm{~nm}) / \mathrm{Py}(4 \mathrm{~nm}) / \mathrm{Cu}(5$ $\mathrm{nm}) / \mathrm{Nb}(20 \mathrm{~nm})(\mathrm{PSV}-\mathrm{B})$ in a constant in-plane field of $5 \mathrm{mT}$. In (b) and (d) the black lines are a guide to the eye. applied field angle $\theta$ (for a $5 \mathrm{mT}$ field) was also investigated and $T_{C}$ was found to be constant for all values of $\theta$.

Before concluding that the trend of $T_{C}$ on $\theta$ can be explained on the basis of spin-triplet pair generation, we must first rule out the possibility that fringing fields from the PSV are suppressing $T_{C}$ (a particular issue in F-S-F structures $[35,36]$ ) or that the proximity effect is due to singlet pairs.

Stray magnetic fields from Py are unlikely since the applied field of $5 \mathrm{mT}$ during the measurement of $T_{C}$ is larger than the minimum or maximum coercive fields of Py (and so the Py is approximately a single domain). The Co is also unlikely to be affected by a $5 \mathrm{mT}$ field since the coercive field is larger than $10 \mathrm{mT}$ and that the remanent state is isotropic on an applied field angle. Nevertheless, to rule out stray fields we repeated steps $1-4$ above on the $\mathrm{Cu} / \mathrm{Py}(4 \mathrm{~nm}) / \mathrm{Cu} / \mathrm{Nb}(20 \mathrm{~nm})$ control sample but with a magnetic field of $0.5 \mathrm{mT}$. In the absence of a second $\mathrm{F}$ layer ( $\mathrm{Co}$ ) spin-one triplet pairs should not be generated and so singlet pairs should dominate the $T_{C}$ of this reference structure. The important $R(T)$ curves corresponding to $H_{a}$ parallel and perpendicular to HA of Py are shown in Fig. 4. Additional $R-T$ data for $H_{a}$ applied $30^{\circ}$ away from the HA of Py are also included. Although $T_{C}$ is weakly affected by an applied field angle, $T_{C}$ is lower by $10 \mathrm{mK}$ (at best) when $H_{a}$ is parallel to the HA, which is the exact opposite trend to that shown in Fig. 3 where $T_{C}$ is lower in the EA configuration. This behavior can be easily understood by considering domain walls in Py: In the HA, $M_{R}$ (Fig. 1) is close to zero implying the domains are randomly orientated with a large domain wall density meaning significant flux must be interacting with $\mathrm{Nb}$ and suppressing its $T_{C}$; in the EA, $M_{R}$ is close to 1 meaning the Py layer is approximately a single domain and so the density of domain walls present must be negligible compared to the HA orientation-hence $T_{C}$ is higher. This analysis rules out a stray field suppression of $T_{C}$ in Fig. 3 and thus supports our hypothesis that the suppression and subsequent minima in $T_{C}$ at $90^{\circ}$ (EA) are related to spin-one triplet pairs enhancing the proximity effect (detected via a suppression of $T_{C}$ ).

To compare Fig. 3 to spin-triplet theory, we calculated the dependence of $T_{C}$ on $\theta$ by numerically solving the linearized Usadel equation [37]. As shown by the solid (pink) curves in Fig. 3, there is a very good qualitative agreement between our model and the experimental results, giving both similar values for $\Delta T_{C}$ and a minimum in $T_{C}$ near $\theta=90^{\circ}$. Identical conductivities and interfacial resistance values are used in both fits-it should be noted that these parameters mostly influence the absolute values of $T_{C}$ and only weakly affect the trend of $T_{C}$ on $\theta$. The two adjustable parameters are the critical temperature of $\mathrm{Nb}\left(T_{C 0}\right)$ in the absence of the PSV and the ratio of the exchange energies of Py and Co. The latter is the most important parameter controlling $T_{C}(\theta)$ and good fits were acquired using the Curie temperatures of Py and Co of $600 \mathrm{~K}$ and $1350 \mathrm{~K}$, respectively.

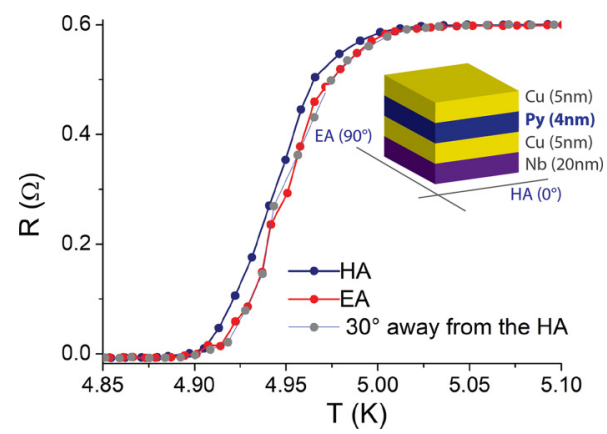

FIG. 4. (Color online) $R(T)$ curves from a $\mathrm{Cu} / \mathrm{Py}(4) / \mathrm{Cu} / \mathrm{Nb}(20)$ (numbers in nm units) control sample with an in-plane magnetic field of $0.5 \mathrm{mT}$ applied along the EA (red) and HA (blue) of Py and also $30^{\circ}$ away from the HA of Py (gray). 
The most notable discrepancy between the theory and experiment is the predicted value of $T_{C}$ for PSV-B in the AP state. This is not necessarily surprising and can be understood as follows: The Usadel equation is strictly applicable in the diffusive limit where the average electron mean free path in the PSV is smaller than any other length scale (e.g., coherence length, spin diffusion length) involved in the problem. Although this condition is likely to be mostly fulfilled since the devices contain many layers and interfaces, the thicknesses of the $\mathrm{F}$ and $\mathrm{N}$ layers are close to their known mean free path lengths and therefore one can expect certain electron trajectories that are almost ballistic. As shown in Ref. [38], in the ballistic limit and in the AP state the net exchange field averages out and hence the ballistic trajectories hardly contribute to the suppression of $T_{C}$. This would explain why for PSV-B with the thinnest Co layer $(3 \mathrm{~nm})$ in the AP state the $T_{C}$ measured is larger than predicted by the Usadel equation. While for PSV-A the Co layer is much thicker (6 nm), meaning the diffusive limit is more applicable.

Finally, returning to the potential for active control of spintronic devices using triplet pairs, the large values of $\Delta T_{C} / T_{C}$ obtained demonstrate a sensitivity of triplet pair generation to the magnetization configuration of the PSV. By extension, integrating similar active components into the barrier of a Josephson junction should lead to large changes in spin (triplet) current density, which is an essential ingredient for the development of superconducting spintronics.

The work was funded by the Royal Society, the Leverhulme Trust through an International Network Grant and the European Research Council (AIG “Superspin”).
[1] A. Fert, Rev. Mod. Phys. 80, 1517 (2008).

[2] H. Yang, S. H. Yang, S. Takahashi, S. Maekawa, and S. S. P. Parkin, Nat. Mater. 9, 586 (2010).

[3] C. H. L. Quay, D. Chevallier, C. Bena, and M. Aprili, Nat. Phys. 9, 84 (2013).

[4] M. Eschrig, Phys. Today 64, 43 (2011).

[5] F. S. Bergeret, A. F. Volkov, and K. B. Efetov, Phys. Rev. Lett. 86, 4096 (2001).

[6] F. S. Bergeret, A. F. Volkov, and K. B. Efetov, Rev. Mod. Phys. 77, 1321 (2005)

[7] M. Houzet and A. I. Buzdin, Phys. Rev. B 76, 060504(R) (2007).

[8] A. I. Buzdin, Rev. Mod. Phys. 77, 935 (2005).

[9] Y. Blum, A. Tsukernik, M. Karpovski, and A. Palevski, Phys. Rev. Lett. 89, 187004 (2002).

[10] V. Shelukhin, A. Tsukernik, M. Karpovski, Y. Blum, K. B. Efetov, A. F. Volkov, T. Champel, M. Eschrig, T. Lofwander, G. Schon, and A. Palevski, Phys. Rev. B 73, 174506 (2006).

[11] J. W. A. Robinson, S. Piano, G. Burnell, C. Bell, and M. G. Blamire, Phys. Rev. Lett. 97, 177003 (2006).

[12] A. A. Bannykh, J. Pfeiffer, V. S. Stolyarov, I. E. Batov, V. V. Ryazanov, and M. Weides, Phys. Rev. B 79, 054501 (2009).

[13] C. Bell, R. Loloee, G. Burnell, and M. G. Blamire, Phys. Rev. B 71, 180501 (2005).

[14] J. W. A. Robinson, S. Piano, G. Burnell, C. Bell, and M. G. Blamire, Phys. Rev. B 76, 094522 (2007).

[15] J. W. A. Robinson, Z. H. Barber, and M. G. Blamire, Appl. Phys. Lett. 95, 192509 (2009).

[16] M. A. Khasawneh, W. P. Pratt, and N. O. Birge, Phys. Rev. B 80, 020506(R) (2009).

[17] S. Piano, J. W. A. Robinson, G. Burnell, and M. G. Blamire, Eur. Phys. J. B 58, 123 (2007)

[18] J. W. A. Robinson, G. B. Halász, A. I. Buzdin, and M. G. Blamire, Phys. Rev. Lett. 104, 207001 (2010).

[19] T. S. Khaire, M. A. Khasawneh, W. P. Pratt, and N. O. Birge, Phys. Rev. Lett. 104, 137002 (2010).

[20] J. W. A. Robinson, J. D. S. Witt, and M. G. Blamire, Science 329, 59 (2010); G. B. Halász, M. G. Blamire, and J. W. A. Robinson, Phys. Rev. B 84, 024517 (2011); N. Banerjee, C. B. Smiet, R. G. J. Smits, A. Ozaeta, F. S. Bergeret, M. G. Blamire, and J. W. A Robinson, Nat. Commun. 5, 3048 (2014).
[21] M. S. Anwar, F. Czeschka, M. Hesselberth, M. Porcu, and J. Aarts, Phys. Rev. B 82, 100501(R) (2010).

[22] D. Sprungmann, K. Westerholt, H. Zabel, M. Weides, and H. Kohlstedt, Phys. Rev. B 82, 060505(R) (2010).

[23] C. Klose, T. S. Khaire, Y. Wang, W. P. Pratt, Jr., N. O. Birge, B. J. McMorran, T. P. Ginley, J. A. Borchers, B. J. Kirby, B. B. Maranville, and J. Unguris, Phys. Rev. Lett. 108, 127002 (2012).

[24] Y. Wang, W. P. Pratt, Jr., and N. O. Birge, Phys. Rev. B 85 , 214522 (2012).

[25] M. S. Anwar, M. Veldhorst, A. Brinkman, and J. Aarts, Appl. Phys. Lett. 100, 052602 (2012).

[26] J. W. A. Robinson, F. Chiodi, M. Egilmez, G. B. Halász, and M. G. Blamire, Sci. Rep. 2, 699 (2012).

[27] J. D. S. Witt, J. W. A. Robinson, and M. G. Blamire, Phys. Rev. B 85, 184526 (2012).

[28] Y. Kalcheim, T. Kirzhner, G. Koren, and O. Millo, Phys. Rev. B 83, 064510 (2011)

[29] Y. Kalcheim, O. Millo, M. Egilmez, J. W. A. Robinson, and M. G. Blamire, Phys. Rev. B 85, 104504 (2012).

[30] C. Visani, Z. Sefrioui, J. Tornos, C. Leon, J. Briatico, M. Bibes, A. Barthélémy, J. Santamaría, and Javier E. Villegas, Nat. Phys. 8, 539 (2012).

[31] J. Linder, T. Yokoyama, and A. Sudbø, Phys. Rev. B 79, 224504 (2009).

[32] N. G. Pugach and A. I. Buzdin, Appl. Phys. Lett. 101, 242602 (2012).

[33] Y. V. Fominov, A. A. Golubov, T. Y. Karminskaya, M. Y. Kupriyanov, R. G. Deminov, and L. R. Tagirov, JETP Lett. 91, 308 (2010).

[34] P. V. Leksin, N. N. Garif'yanov, I. A. Garifullin, Y. V Fominov, J. Schumann, Y. Krupskaya, V. Kataev, O. G. Schmidt, and B. Buchner, Phys. Rev. Lett. 109, 057005 (2012).

[35] R. Steiner and P. Ziemann, Phys. Rev. B 74, 094504 (2006).

[36] J. Zhu, X. Cheng, C. Boone, and I. N. Krivorotov, Phys. Rev. Lett. 103, 027004 (2009).

[37] See Supplemental Material at http://link.aps.org/supplemental/ 10.1103/PhysRevB.89.140508 for details concerning the numerical calculations and fits to the experimental data.

[38] Y. M. Blanter and F. W. J. Hekking, Phys. Rev. B 69, 024525 (2004). 